\title{
CrimRxiv
}

\section{Preventing Bullying Through Science, Policy, and Practice}

National Research Council

Published on: May 10, 2016

DOI: $10.21428 / c b 6 a b 371.8 b 0 e c 66 c$

License: Creative Commons Attribution 4.0 International License (CC-BY 4.0). 
